\title{
VHDL IMPLEMENTATION FOR EDGE DETECTION USING LOG GABOR FILTER FOR DISEASE DETECTION
}

\author{
V.V.Kumbhalwar ${ }^{1}$, S.R.Dixit ${ }^{2}$ \\ ${ }^{I}$ M.Tech Student, Communication Engineering, Department of Electronics \& Telecommunication Engineering, \\ G.H.Raisoni College of Engineering \& Technology, Nagpur-440016, India. \\ kumbhalwar_vipul.ghrcemtechcom@raisoni.net \\ ${ }^{2}$ Assistant Professor, Department of Electronics \& Telecommunication Engineering, G.H.Raisoni College of \\ Engineering \& Technology, Nagpur-440016, India. \\ swati.dixit@raisoni.net
}

\begin{abstract}
Edge detection is first and essential step in the field of image processing. Detected edges play a very important role such as image enhancement, object detection, focus area selection and many more. In medical application like tonsillitis, tumor, fracture can be detected in its early stage by detecting edges of disease. There are different and many ways for edge detection, However, the most may be grouped into three categories, first order gradient, second order and optimal edge detection.. Sobel edge detection is gradient based edge detection method used for finding edges of image. Also Sobel edge detection method provide one more advantage that it having better noise sensitivity as compared to other edge detection method. Here new concept Log-Gabor filter is used for best contrast ridges, efficient noise reduction and improved edges of an images. Most image processing tools such LabVIEW are not suited for strong real-time constraints, so to overcome this problem hardware implementation FPGA used. Proposed model for disease detection is design in LabVIEW platform with NI Vision Assistant tool 14.0 .
\end{abstract}

Keywords: Edge Detection, Sobel Operator, Log-Gabor Filter, Labview 14.0.

\section{INTRODUCTION}

In medical application, disease like tonsillitis, tumor and many more can be detected in its early stage and can be cured by edge detection. So edges play very important and essential role in image processing[1]. The early stages of image processing identify features in images that are relevant to estimating the properties and also structure of objects in a scene. Edges is nothing but a notable local changes in the image and are important features for analyzing images. Normally edges are occur on the boundary between two different regions in an image. Edge detection is probably frequent and the first basic step in recovering information from images. Due to its importance, edge detection continues to be an active research area[3]. Quality of detected edges use in image segmentation, scene analysis, object recognition. For accurate edge extraction, both changes in the color and changes in the brightness between neighboring pixels should be examined.

Edge detection are broadly classified into three methods[2]

1. First order derivative (Gradient Method) Method.

- Sobel Operator

- Prewitt Operator

- Robert Operator

2. Second order derivative Method.

- Laplacian

- Laplace of Gaussian
- Difference of Gaussian

3. Optimal edge detection method.

- Canny edge detection.

Here Sobel operator based edge detection technique used and extended it for real-time applications. Due to its property to counteract the noise sensitivity, Sobel operator edge detection over other gradient operators are used. The sobel operator uses two $3 \times 3$ matrix parameter and kernels which are with the original image to calculate approximations of the derivatives one for horizontal changes in an image, and second one for vertical changes in that image.

In this scenario for better noise sensitivity, best contrast ridges and improved edges of an image Log-Gabor filter used. Log-Gabor filter having two characteristics which is the reason we use it in image processing. firstly, Log-Gabor functions, always have zero DC component, means it work with no dc component, it help to improve improve not only the contrast ridges but also improve edges of images. Secondly, the transfer function of the Log-Gabor function has an extended tail at the high frequency end, that oftenly enables one to obtain a very wide spectral information with localized spatial extent and consequently helps to preserve true ridge structures of images[1]. Disease detection model script first design in NI Vision Assistant which is image processing tool used in LabVIEW. After the completion of 
script, it export on LabVIEW software so that we get LabVIEW code. Sometime in the field of medical, hardware basis system is more preferable than software and result is also efficient as hardware comes along with software, so entire disease detection design implement on FPGA to get accurate disease detection. LabVIEW FPGA tool is preferred so that entire disease detection design can be easily implement to FPGA Flexrio board.
The proposed title aims to design disease detection system for detection of tonsillitis disease in medical field. The below block diagram in fig. 1 shows the proposed system for disease detection using sobel operator and Log-Gabor filter. Proposed model is design for real time processing as it consist of an camera. Capture image forward to LabView FPGA tool for detection of disease and after processing in LabVEW result can determined.

\section{BLOCK DIAGRAM OF PROPOSED SYSTEM}

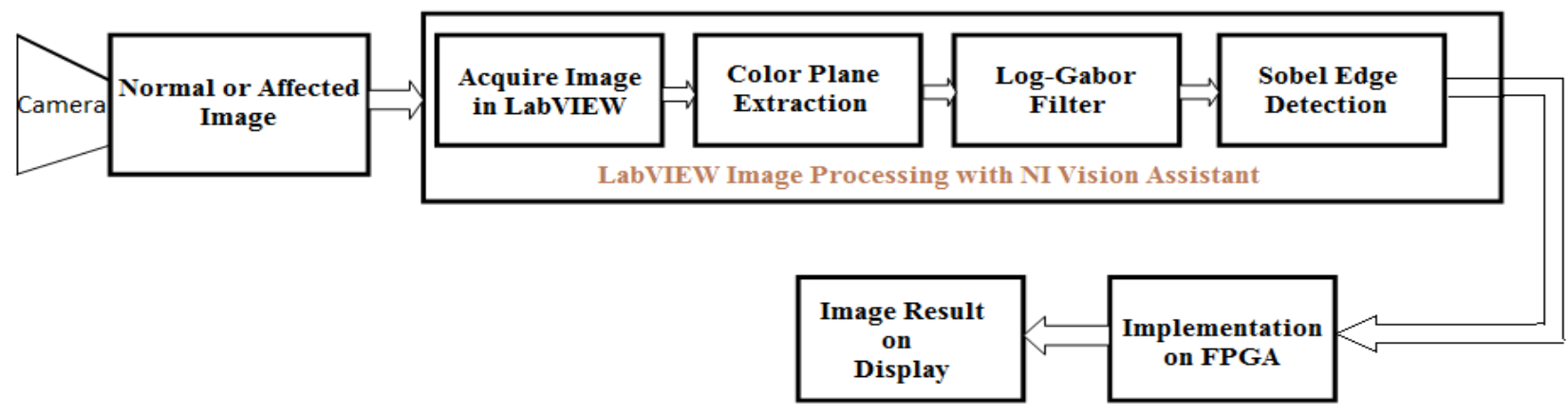

Fig-1: Proposed disease detection model

Camera: Camera capture the input image it may be normal or disease affected and it is further processed.

Color plane extraction: To convert color image into grayscale image here color plane extraction used. It improve the ridges of image. The result of color plane extraction left us with a grayscale image that gives bright intensities for strong edges, low intensities for weak edges, and gives black portion for no ridges.

Log-Gabor filter: Log-Gabor filter improve contrast ridges as well as reduce noise from images. By using Log-Gabor filter we can work on more than one octave bandwidth. Filter used for noise reduction purpose from grayscale image.

Sobel Operator: Sobel operator is gradient method and it is the best edge detection method commonly used in image processing, as it gives efficient edges of an image. In Sobel operator two $3 \times 3$ filters $\mathrm{Hx}$ and Hy are mainly used. 3x3 spatial mask which are convolved with the original image to calculate the approximation of the gradient. In below matrix $\mathrm{Hx}$ is the convolution kernel in $\mathrm{x}$ direction and $\mathrm{Hy}$ is the convolution kernel for $\mathrm{y}$ direction.

$\mathrm{Hx}=\left[\begin{array}{ccc}-1 & 0 & 1 \\ -2 & 0 & 2 \\ -1 & 0 & 1\end{array}\right]$

$\mathrm{Hy}=\left[\begin{array}{ccc}-1 & -2 & -1 \\ 0 & 0 & 0 \\ 1 & 2 & 1\end{array}\right]$ correspondingly. The local edge strength is defined as the gradient magnitude given by,

$\mathrm{GM}(\mathrm{x}, \mathrm{y})=\sqrt{2(H x)+2(H y)}$

Gradient magnitude of image defines local edge strength and is given by equation,

$\mathrm{GM}(\mathrm{x}, \mathrm{y})=|\mathrm{Hx}|+|\mathrm{Hy}|$

Implementation on FPGA: The salient feature of FPGAs that makes them superior in speed. Real time image processing is possible with FPGA. When any software comes with hardware then performance for that system increase.

\section{COLOR PLANE EXTRACTION INLABVIEW}

Figure 2 represent color plane extraction by using LabVIEW software. Color image is the combination of three basic color, red, green and blue. We can stack the three planes together, like a sandwich. In the RGB color model, the first plane corresponds to the Red plane, the second to the Green plane and the third to the Blue plane. After stacking the planes together we have to indicate that the image will be using the RGB Color Model. This code is designed to show how to manipulate a RGB image to show the different color planes as well as how to grayscale an image.

The gradient paremeter across the neighboring lines or neighboring columns are computed by these filter 


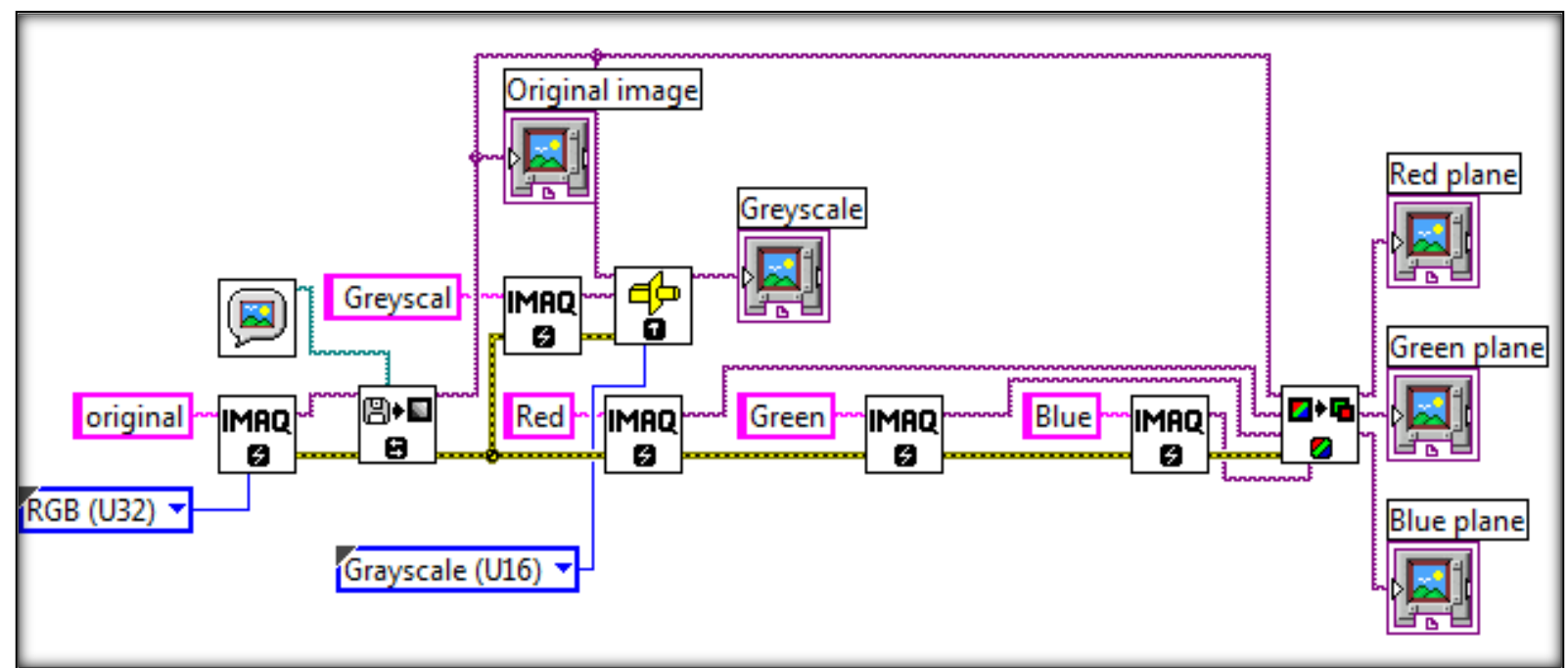

Fig-2: Color Plane Extraction code using LabVIEW

Figure 3 showing output result of color plane extraction. We are getting grayscale image by extracting any one color from RGB image, only intensity get vary for each color extraction. Ridges for each color extraction is varied according to pixel intensity.

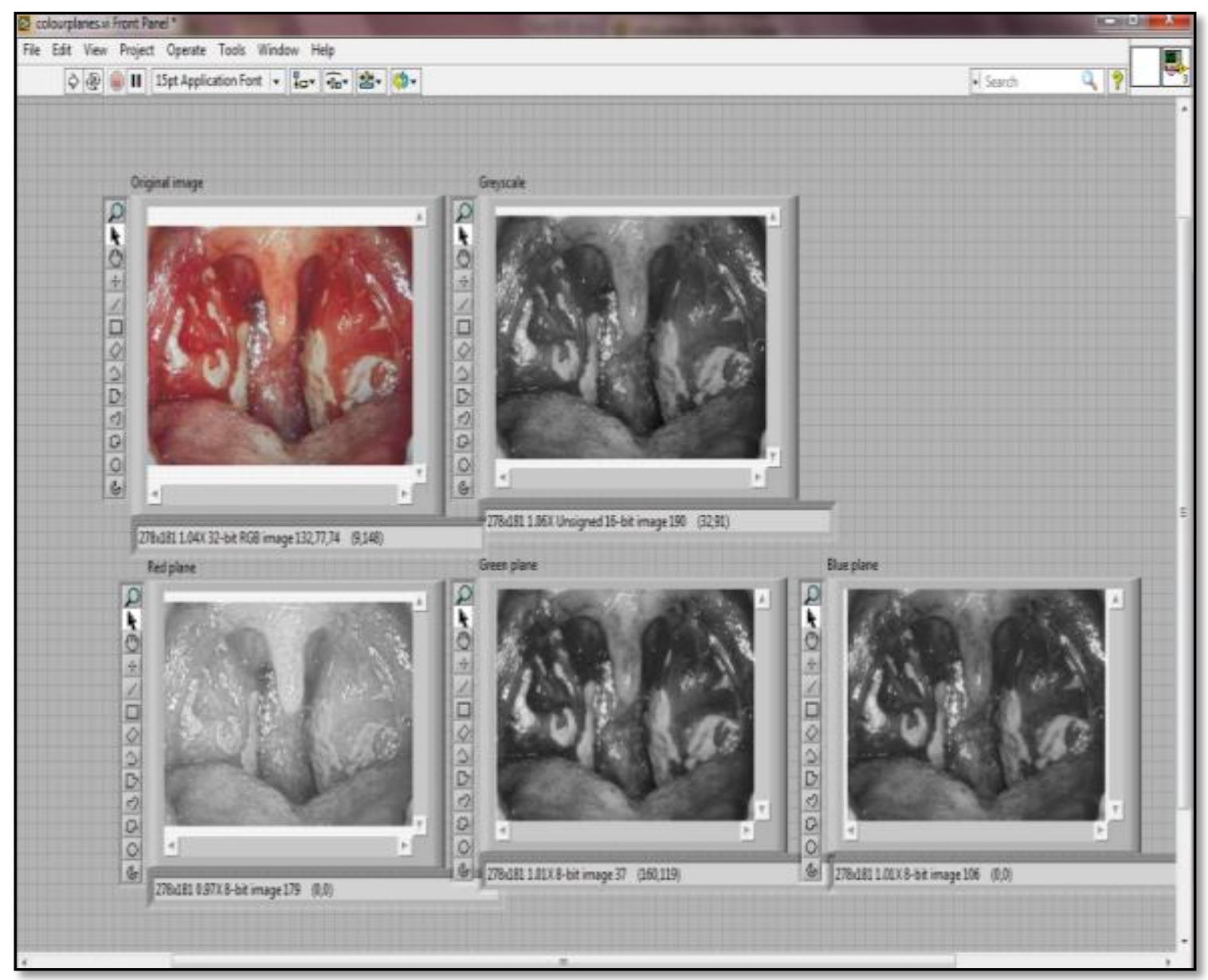

Fig-3: Color plane extraction output on LabVIEW front panel

\section{SOBEL OPERATOR ALGORITHM WITH}

\section{LABVIEW}

Figure 5 showing sobel edge detection algorithm developed in LabVIEW for tonsillitis disease detection. Image acquisition tool is used for image detection and process purpose. File dialog consist total image processing parameter which proposed image preprocessing and filtering operation. 


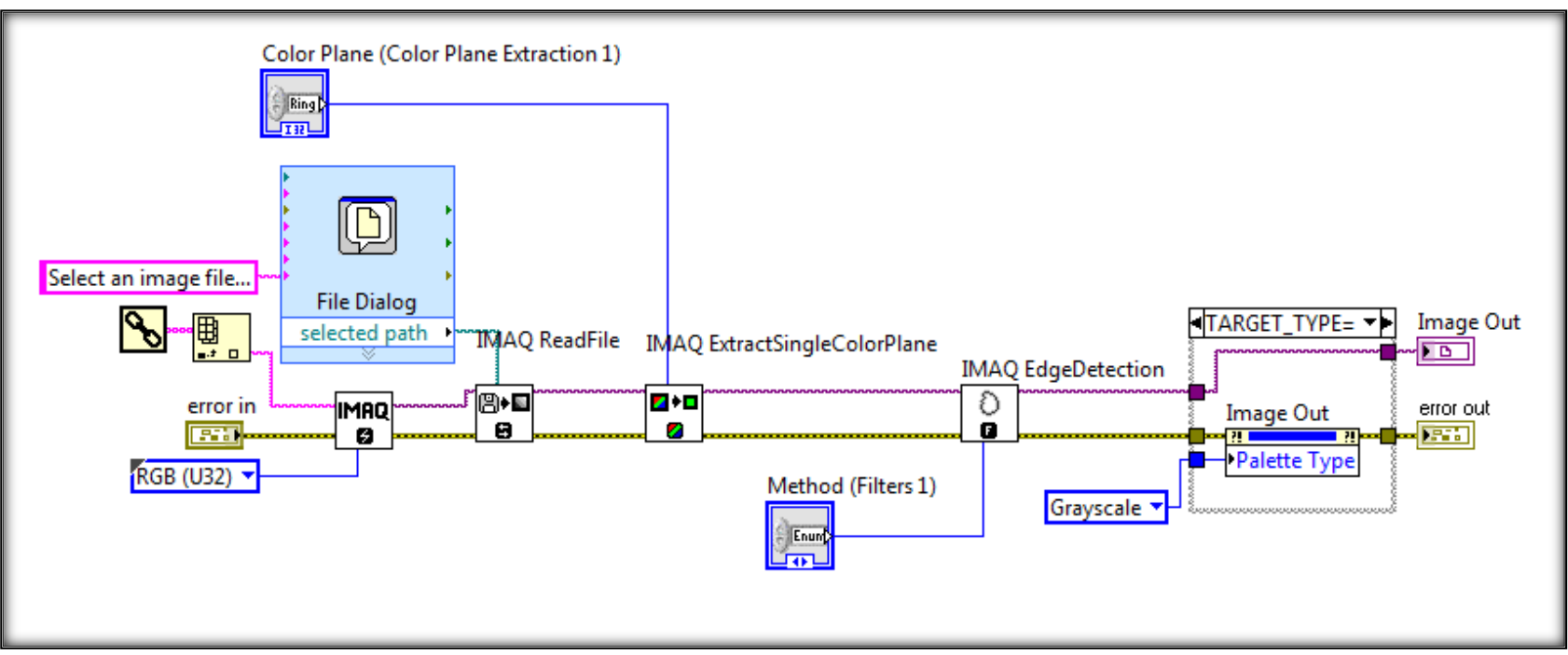

Fig-5: Edge Detected using sobel Operator

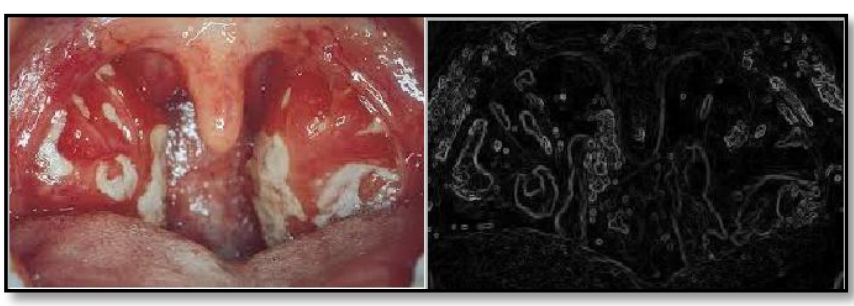

Fig-6 (a):Tonsillitis Image Fig-6 (b):Sobel Edge Detection

\section{RESULT}

Figure 6(a) Showing LabVIEW FPGA design for tonsillitis disease detection and result of the current work. The bright region of disease obtained from using color plane extraction and by using log-gabor filter we get efficient result also by using log-gabor filter system can work more efficiently. Here in this system tonsillitis disease detected.

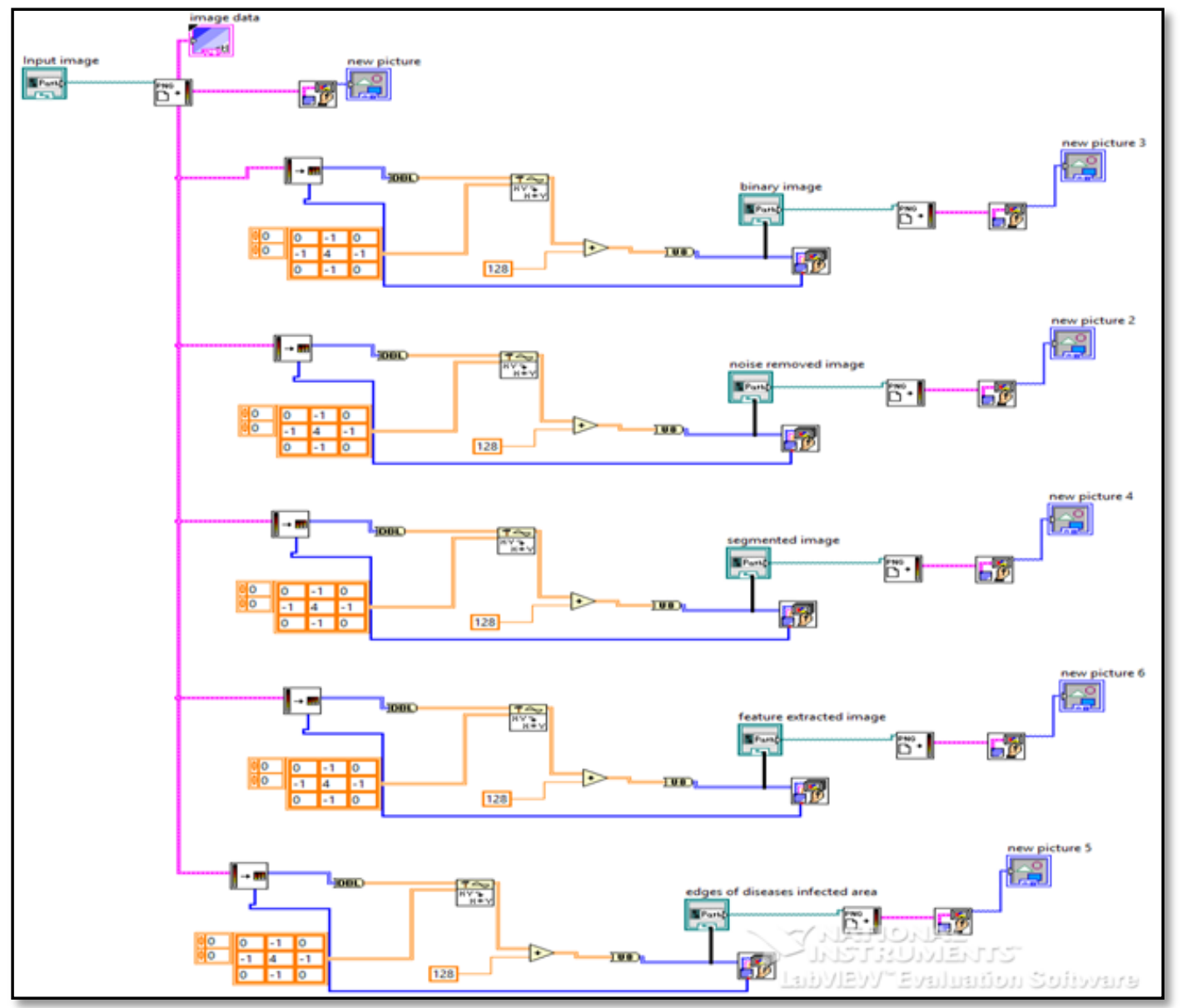

Fig-7(a): LabVIEW design for disease detection 


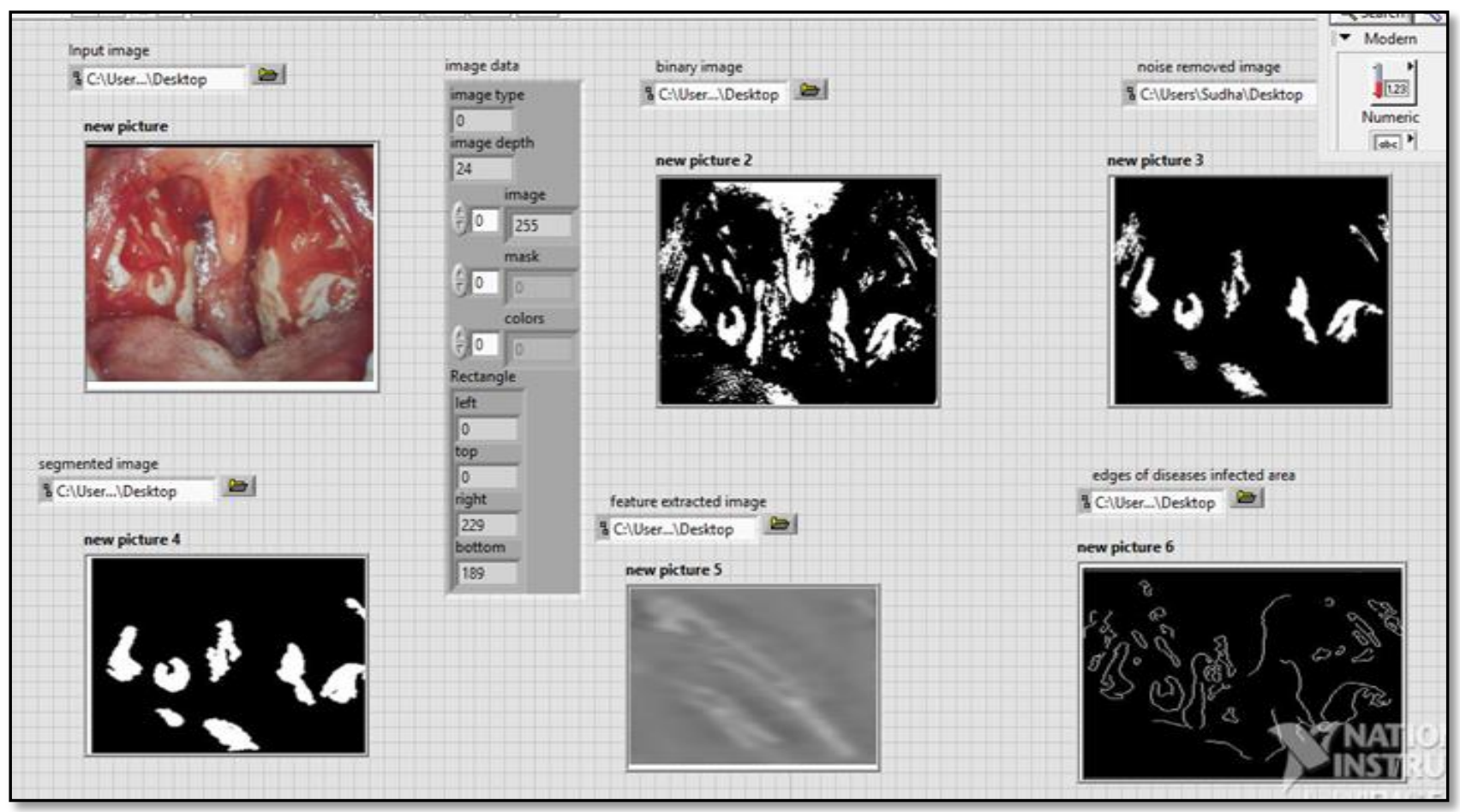

Fig-7 (b): Disease detection results

\section{CONCLUSION}

This paper discusses the design of the sobel edge-detection for tonsillitis images with the help of LabVIEW 14.0 and LabVIEW FPGA tool. Paper conclude that Edge detection using software and hardware is challenging task like total hardware software compatibity. Main advantage of sobel operator is better noise sensitivity and it provide sharp edges as compare to other edge detection method.

\section{REFERENCES}

[1] Bharath K, Dr. N. G. Kurahatti, "VERILOG DESIGN FOR FEATURE EXTRACTION USING LOG-GABOR FILTER FOR DISEASE DETECTION" International Journal For Technological Research In Engineering Volume 2, Issue 9, May-2015.

[2] Aniket A. Ingle, Vrushali G. Raut "Hardware software co-simulation of edge detection for image processing system using delay block in xsg" IJRET: International Journal of Research in Engineering and Technology Volume: 03 Issue: 05 | May-2014.

[3] Rucha R. Thakur, Swati R. Dixit, Dr.A.Y.Deshmukh "VHDL Design for Image Segmentation using Gabor filter for Disease Detection" International Journal of VLSI design \& Communication Systems (VLSICS) Vol.3, No.2, April 2012.

[4] Girish N Chaple, R.D.Daruwala "Comparisons of Robert, Prewitt, Sobel operator based edge detection method for real time uses on FPGA" 2015 international conference on technology for sustainable development Feb04-06-2015.

[5]Kuldeepak, Monoka kaushak, Munish Vashishath "License Plate Recognition System based on Image Processing Using Labview" International Journal of Electronics Communication and Computer Technology (IJECCT) Volume 2 Issue 4 (July 2012).
[6]K. Sudharani, A.swapnarani, K.manikumari,t. C. Sarma. Satya prasad "labview based brain tumor area and length detection in ct and mri scan images" International Journal of Advanced Trends in Computer Science and Engineering (IJATCSE), Vol.2, No.5, Pages :70-74 (2013) Special Issue of ICCECT 2013 - Held during September 20, 2013, Bangalore, India.

[7] Ravi Kumar A.V, Dr. Nataraj K.R, Dr.Rekha K.R "Morphological Real Time Video Edge Detection in Labview" International Journal of Computer Science and Information Technologies, Vol. 3 (2) , 2012,3808-3811.

[8] Sanjay Singh, Anil Kumar Saini, Ravi Saini, A. S. Mandal, Chandra Shekhar,1 and Anil Vohra "Area Optimized FPGA-Based Implementation of The Sobel Compass Edge Detector" Hindawi Publishing Corporation ISRN Machine Vision Volume 2013, Article ID 820216.

[9] Yasri, N.H.Hamid, V.V.Yap "Performance analysis of FPGA based sobel edge detection operator" IEEE.

[10] Ankush R. Bhagat, Swati R. Dixit, Dr. A.Y. Deshmukh "VHDL based Sobel Edge Detection" International Journal of Engineering Research and General Science Volume 3, Issue 1, January-February, 2015.

[11] Christos Kykou,Christos Ttoffis and Theocharis Theocharides, "FPGA-ACCELERATED OBJECT DETECTION USING EDGE INFORMATION", International Conference on Field Programmable Logic and Applications, 2011

[12] J.Canny, "A computational approach to edge detection", IEEE transaction on pattern analysis and machine intelligence, vol 8, no.6, pp,679- 698,Nov 1986.

[13] S. Singh, A.K. Saini, R. Saini, AS Mandal, C. Shekhar, A. Vohra, "Area Optimized FPGA based Implementation of Sobel Compass Edge Detector", ISRN Machine Vision, vol. 2013, Article ID 820216, pp. 6, 2013. 